Revista de Matemática: Teoría y Aplicaciones 2009 16(1) : 76-104

CIMPA - UCR ISSN: 1409-2433

\title{
PREDICCIÓN ESTACIONAL DEL CLIMA EN CENTROAMÉRICA MEDIANTE LA REDUCCIÓN DE ESCALA DINÁMICA. PARTE II: APLICACIÓN DEL MODELO MM5V3
}

\author{
ERICK R. RiverA* JORge A. AMAdOR ${ }^{\dagger}$ \\ Recibido/Received: 20 Feb 2008 - Aceptado/Accepted: 25 Jul 2008
}

\begin{abstract}
Resumen
En la primera parte de este trabajo se determinó que el modelo de circulación general (MCG) ECHAM4.5 posee más habilidad para simular aspectos dinámicos y termodinámicos de la estructura de la atmósfera asociados a las características climáticas de la región de Centroamérica, en comparación con el CCM3.6. Por tal motivo, la información proveniente de este MCG se utilizó para conducir un experimento de reducción de escala dinámica con el modelo regional MM5v3, en el cual se generó un conjunto de simulaciones (realizaciones estadísticas) de alta resolución espacial (30 $\mathrm{km}$ ) para el mes de enero del año 2000.

Los resultados de la reducción de escala dinámica con el MM5v3 permiten establecer que este modelo regional es capaz de reproducir adecuadamente aspectos del clima centroamericano que los MCG no pueden capturar debido a que poseen limitaciones de resolución espacial y a que no representan adecuadamente los rasgos topográficos y las interacciones físicas y dinámicas asociadas a la mesoescala. La comparación con datos derivados de observaciones indica que el MM5v3 simula la región de máximo de viento de bajo nivel que está relacionada con la corriente en chorro de los Mares Intra-Americanos, aunque la intensidad de ésta es subestimada. En cuanto a los patrones de precipitación, éstos coinciden con los obtenidos de las observaciones (seco en el Pacífico, más lluvioso en el Caribe), sin embargo, existe un exceso generalizado en la cantidad de lluvia simulada producto de los esquemas de parametrización utilizados (Grell y Kain-Fritsch). En el análisis de la desviación estándar de la muestra de doce
\end{abstract}

\footnotetext{
* Centro de Investigaciones Geofísicas, Universidad de Costa Rica, 2060 San José, Costa Rica. E-mail: errivera@cariari.ucr.ac.cr

${ }^{\dagger}$ Centro de Investigaciones Geofísicas y Escuela de Física, Universidad de Costa Rica, 2060 San José, Costa Rica. E-mail: jamador@cariari.ucr.ac.cr
} 
miembros, se detectan las áreas en las que este modelo regional tiene mayor dispersión o incertidumbre, entre ellas destaca una localizada principalmente al sur de Panamá.

Palabras clave: modelos numéricos, predicción climática estacional, reducción de escala dinámica, clima, variabilidad climatica.

\begin{abstract}
In the first part of this work it was determined that general circulation model (GCM) ECHAM4.5 shows more ability than CCM3.6 to simulate key climate features of Central America. For such reason, output from ECHAM4.5 was used to perform a dynamical downscaling experiment using the regional model MM5v3, in which a set of high-resolution simulations (of up to $30-\mathrm{km}$ horizontal resolution) was generated for January 2000.

The results of the dynamical downscaling allow to conclude that MM5v3 is able to suitably reproduce aspects of the Central American climate that GCMs cannot capture because of their coarse horizontal resolution, their limitations in representing both the regional topography and the mesoscale dynamical interactions. Comparison with data derived from observations indicates that the MM5v3 simulates the region of maximum low-level wind that is related to the Intra-Americas Seas Low Level Jet, although the regional model underestimates its intensity. Regarding the precipitation patterns, they agree with those derived from the observations (drier areas in the Pacific, wetter areas in the Caribbean). Nevertheless, there is a generalized overestimation in the amount of simulated rain. The analysis of the standard deviation for a twelve-member sample shows areas in which MM5v3 has greater dispersion or uncertainty (mainly to the south of Panama).
\end{abstract}

Keywords: numerical models, seasonal climate prediction, dynamical downscaling, climate, climate variability.

Mathematics Subject Classification: 86A10.

\title{
1 Introducción
}

El presente artículo es la segunda parte de un trabajo complementario (Rivera y Amador, 2008, en adelante RA1 en el que se evalúan dos modelos de circulación general (MCG), el ECHAM4.5 y el CCM3.6 (ver descripción y discusión de propiedades usadas en RA1) con el propósito de identificar cual de estos modelos captura mejor los razgos climatológicos en la región cercana a Centroamérica a fin de realizar un proceso de reducción de escala dinámica con el modelo MM5v3. Este proceso, las condiciones utilizadas, los resultados y limitaciones encontradas se presentan en este artículo, denominado de ahora en adelante RA2. El objetivo general de RA2 es brindar una mayor comprensión del problema de la predicción climática a partir de modelos numéricos y su importancia para la región centroamericana, de manera que se pueda dar a conocer la relevancia que tiene el desarrollo de un proceso de evaluación de MCG utilizados en la elaboración de predicciones climáticas y así identificar aquellos que proveen una representación más realista de las características del clima regional. Además, de manera específica se pretende establecer las bases para un método de trabajo que permita la realización de predicciones climáticas de alta resolución 
mediante el uso de modelos numéricos regionales o de área limitada anidados en MCG que proporcionen la mejor información de gran escala (proceso de reducción de escala dinámica).

En este trabajo, se utiliza el modelo regional MM5v3 para efectuar una reducción de escala dinámica y obtener simulaciones climáticas para el mes de enero de 2000 inicializadas con datos del modelo global ECHAM4.5 y con una resolución espacial de hasta $30 \mathrm{~km}$.

\section{El problema de la reducción de escala y el modelo MM5v3}

\subsection{Reducción de escala dinámica}

Los modelos climáticos globales poseen resoluciones del orden de cientos de kilómetros o más. Takle et al. (1999) afirman que aunque esto es útil para diferentes propósitos, no es suficiente para simular circulaciones que influencian el clima a escala regional. Es por ello que se han desarrollado técnicas que permiten tomar la información proveniente de modelos globales y aplicarla a una región específica. Estas aplicaciones dinámicas se conocen como reducción de escala o downscaling (Chase et al., 2003).

Básicamente, existen dos tipos de técnicas de reducción de escala: (a) estadística, la cual usa relaciones históricas y empíricas de índole matemática entre los valores de gran escala y las condiciones en una cierta área, y (b) dinámica, la cual hace uso de un modelo numérico regional (MNR) para generar información climática regional que sea realista y que guarde consistencia física y dinámica con los patrones de circulación de gran escala que son provistos ya sea por datos observados o por un MCG (Chase et al., 2003; Leung et al., 2003; Castro et al., 2005; Wang et al., 2004). Dickinson et al. (1989) y Giorgi y Bates (1989) realizaron las primeras demostraciones de modelado climático regional usando métodos numéricos y a partir de ahí se han dedicado grandes esfuerzos para su desarrollo, aplicación y evaluación (Wang et al., 2004).

Particularmente, en el caso de la reducción de escala dinámica, el área limitada de los MNR permite llevar a cabo simulaciones de meses y hasta años a resoluciones de decenas de kilómetros (Takle et al., 1999). Dada esta situación, es posible obtener mejores representaciones de las circulaciones atmosféricas, el comportamiento de las variables dinámicas

y termodinámicas y los efectos en el clima regional debidos, por ejemplo, a la topografía y a líneas costeras complejas (Giorgi, 1990; Jones et al,. 1995; Walsh and McGregor, 1995; Hesselbjerg, 2000; Wang et al., 2004).

De acuerdo con Chase et al. (2003), un aspecto importante a considerar en las técnicas estadísticas y dinámicas de reducción de escala es que primeramente se formula la suposición de que la información de gran escala es confiable y precisa. Si el modelo de gran escala proporciona información incorrecta, ninguna de las dos técnicas puede corregirla. Esta situación establece la necesidad de comparar las simulaciones climáticas de los MCG con datos observados, es decir, realizar un proceso de evaluación antes de efectuar una reducción de escala.

Según Wang et al. (2004), muchos estudios sugieren que los MCG con mayor resolución espacial proveen mejores simulaciones de gran escala (por ejemplo, Pope y Stratton, 2002; Duffy et al., 2003). Además, estos autores indican que la evaluación debe ayudar a selec- 
cionar los MCG que produzcan resultados más realistas para el área de estudio y también debe brindar claridad en cuanto a la mejor configuración del dominio del MCR, con el fin de evitar la colocación de los contornos laterales sobre áreas en donde se ha detectado que el MCG tiene problemas; por ejemplo, en una zona en donde existe un mal posicionamiento de características de gran escala como la ZCIT; sin embargo, esto no es siempre posible.

Según Wilby y Dawson (2001), las principales fortalezas y debilidades del proceso de reducción de escala dinámica son las siguientes:

1. Fortalezas:

a) Permite obtener información climática con resoluciones de 10 - $50 \mathrm{~km}$ a partir de la salida de un MCG cuyas resoluciones espaciales son del orden de 200 o más kilómetros.

b) Responde a los diferentes forzamientos externos de manera físicamente consistente en contraste con una simple interpolación matemática.

c) Resuelve procesos atmosféricos tales como la precipitación orográfica y procesos físicos de mesoescala.

d) Guarda consistencia en su interacción con el MCG.

2. Debilidades:

a) Depende del realismo del forzamiento de contorno del MCG.

b) La escogencia del tamaño del dominio y su ubicación afecta los resultados.

c) Requiere importantes recursos de cómputo.

d) Las condiciones de contorno iniciales afectan los resultados.

e) La escogencia del esquema de convección/nubes y su consistencia con los del MCG afecta los resultados (especialmente de la precipitación).

\section{$2.2 \quad$ El MM5v3 y el sistema primitivo de ecuaciones}

De acuerdo con Dudhia et al. (2005), las siguientes son las ecuaciones para las variables básicas del modelo no hidrostático en términos de las coordenadas que siguen la forma del terreno $(x, y, \sigma)$.

Presión:

$$
\frac{\partial p^{\prime}}{\partial t}-\rho_{0} g w+\gamma p \vec{\nabla} \cdot \vec{V}=-\vec{V} \cdot \vec{\nabla} p^{\prime}+\frac{\gamma p}{T}\left(\frac{\dot{Q}}{c_{p}}+\frac{T_{0}}{\theta_{0}} D_{\theta}\right)
$$

Momento (componente en $x)$ :

$\frac{\partial u}{\partial t}+\frac{m}{\rho}\left(\frac{\partial p^{\prime}}{\partial x}-\frac{\sigma}{p^{*}} \frac{\partial p^{*}}{\partial x} \frac{\partial p^{\prime}}{\partial \sigma}\right)=-\vec{V} \cdot \vec{\nabla} u+v\left(f+u \frac{\partial m}{\partial y}-v \frac{\partial m}{\partial x}\right)-e w \cos \alpha-\frac{u w}{r_{\text {tierra }}}+D_{u}$ 
Momento (componente en $y)$ :

$\frac{\partial v}{\partial t}+\frac{m}{\rho}\left(\frac{\partial p^{\prime}}{\partial y}-\frac{\sigma}{p^{*}} \frac{\partial p^{*}}{\partial y} \frac{\partial p^{\prime}}{\partial \sigma}\right)=-\vec{V} \cdot \vec{\nabla} v-u\left(f+u \frac{\partial m}{\partial y}-v \frac{\partial m}{\partial x}\right)-e w \sin \alpha-\frac{v w}{r_{\text {tierra }}}+D_{v}$

Momento (componente en $z$ ):

$\frac{\partial w}{\partial t}+\frac{\rho_{0}}{\rho} \frac{g}{p^{*}} \frac{\partial p^{\prime}}{\partial \sigma}+\frac{g}{\gamma} \frac{p^{\prime}}{p}=-\vec{V} \cdot \vec{\nabla} w+g \frac{p_{0}}{p} \frac{T^{\prime}}{T_{0}}-\frac{g R_{d}}{c_{p}} \frac{p^{\prime}}{p}+e(u \cos \alpha-v \sin \alpha)+\frac{u^{2}+v^{2}}{r_{\text {tierra }}}+D_{w}$

Termodinámica:

$$
\frac{\partial T}{\partial t}=-\vec{V} \cdot \vec{\nabla} T+\frac{1}{\rho c_{p}}\left(\frac{\partial p^{\prime}}{\partial t}+\vec{V} \cdot \vec{\nabla} p^{\prime}-\rho_{0} g w\right)+\frac{Q}{c_{p}}+\frac{T_{0}}{\theta_{0}} D_{\theta}
$$

Términos de advección pueden ser expresados como:

$$
\vec{V} \cdot \vec{\nabla} A \equiv m u \frac{\partial A}{\partial x}+m v \frac{\partial A}{\partial y}+\dot{\sigma} \frac{\partial A}{\partial \sigma}
$$

donde,

$$
\dot{\sigma}=-\frac{\rho_{0} g}{p^{*}} w-\frac{m \sigma}{p^{*}} \frac{\partial p^{*}}{\partial x} u-\frac{m \sigma}{p^{*}} \frac{\partial p^{*}}{\partial y} v
$$

Términos de divergencia pueden ser expresados como:

$$
\vec{\nabla} \cdot \vec{V}=m^{2} \frac{\partial}{\partial x}\left(\frac{u}{m}\right)-\frac{m \sigma}{p^{*}} \frac{\partial p^{*}}{\partial x} \frac{\partial u}{\partial \sigma}+m^{2} \frac{\partial}{\partial y}\left(\frac{v}{m}\right)-\frac{m \sigma}{p^{*}} \frac{\partial p^{*}}{\partial y} \frac{\partial v}{\partial \sigma}-\frac{\rho_{0} g}{p^{*}} \frac{\partial w}{\partial \sigma}
$$

Símbolos:

$c_{p}$ : Calor específico a presión constante,

$e: 2 \Omega \cos \lambda$, donde $\lambda$ es la latitud,

$f:$ Parámetro de Coriolis,

$g$ : Aceleración de la gravedad,

$m$ : Factor de escala,

$p$ : Presión,

$p^{*}$ : Presión en superficie menos presión en el tope del modelo,

$p^{\prime}$ : Perturbación de la presión,

$p_{0}$ : Presión de referencia,

$r_{\text {tierra }}$ : Radio medio de la Tierra,

$t$ : Tiempo,

$u$ : Componente zonal del viento,

$v$ : Componente meridional del viento,

$w$ : Velocidad vertical,

$x$ : Coordenada $x$,

$y$ : Coordenada $y$,

$A$ : Variable cualquiera, 
$D_{i}$ : Términos de difusión horizontal y vertical o de mezcla vertical debido a turbulencia en la capa límite planetaria,

$Q$ : Término no adiabático,

$R_{d}$ : Constante del gas para aire seco,

$T$ : Temperatura,

$T^{\prime}$ : Perturbación de la temperatura,

$T_{0}$ : Temperatura de referencia,

$\vec{V}$ : Vector viento,

$\alpha: \phi-\phi_{c}$, donde $\phi$ es la longitud y $\phi_{c}$ es la longitud central,

$\theta_{0}$ : Temperatura potencial de referencia,

$\gamma$ : Razón de capacidades caloríficas $c_{p} / c_{v}$ para aire seco,

$\rho$ : Densidad del aire,

$\rho_{0}$ : Densidad de referencia,

$\sigma$ : Coordenada vertical $\sigma$.

El MM5v3 representa la quinta generación del modelo de mesoescala desarrollado en Pennsilvania State University (PSU) y el National Center for Atmospheric Research (NCAR). Grell et al. (1993), al igual que trabajos de Cox et al. (1998) y Silva (2000), presentan descripciones generales del modelo MM5v3.

Este modelo, de pronóstico del tiempo, está basado en las ecuaciones primitivas (Gill, 1992; Grell et al., 1993; Dudhia et al., 2005, RA1) e incorpora la condición no hidrostática, a diferencia de su antecesor, el MM5v2. Utiliza coordenadas verticales sigma $\left(\sigma=p / p_{0}\right.$; donde $p$ es la presión atmosférica en un nivel dado y $p_{0}$ la presión atmosférica en superficie) y resuelve las ecuaciones mediante el método de diferencias finitas. Tiene capacidad para anidar múltiples dominios, con una relación de transformación de la resolución de 3:1 y puede realizar interacciones de 2 vías (2-way nesting), o sea, retroalimentaciones entre los dominios madre (externos) y los dominios anidados (internos). La representación física de la capa límite planetaria puede ser una parametrización simple de baja resolución vertical que tiene dos regímenes de estabilidad: estable e inestable (bulk parameterization) o una parametrización más detallada basada en una revisión del modelo de capa límite de Blackadar (Zhang y Anthes, 1982) que tiene cuatro regímenes de estabilidad: estable, turbulencia mecánicamente conducida, convección forzada y convección libre. La opción de radiación atmosférica posee esquemas de onda corta y onda larga que interactúan con la superficie y con la atmósfera, incluyendo las nubes y la precipitación (Dudhia, 1989).

En el MM5v3 se incluyen módulos de precipitación de gran escala y convectiva. Los procesos de gran escala se resuelven de manera explícita. Las opciones de convección de cúmulos profunda incluyen, entre otras, las parametrizaciones basadas en Kuo (1974) y un esquema modificado de Arakawa-Schubert (1974) que incluye flujos descendentes húmedos de escala convectiva.

En este trabajo se emplean dos diferentes tipos de representaciones convectivas, el de Grell (G, Grell et al., 1991) y el de Kain-Fritsch (KF, Kain \& Fritsch, 1993), los cuales se discuten a continuación. El esquema de convección de cúmulos $\mathrm{G}$ es un esquema en el que se considera la razón de cambio en el tiempo de la estabilidad como principal disparador convectivo. Además, debe existir flotabilidad positiva y se tiene que considerar la 
intensidad de la cap (región de flotabilidad negativa debajo de un nivel existente de convección libre en donde se debe proveer energía a la parcela para que ésta pueda mantener su movimiento ascendente) con el fin de determinar a qué nivel de altura debe levantarse la parcela para alcanzar su nivel de convección libre. En cuanto a las características de la convección, este esquema utiliza modelos simples de ascensos y descensos para simular los reacomodos de masa producidos por la misma, la eficiencia de evaporación es función de la intensidad de la cortante de viento (únicamente sobre el océano) y toda el agua de nube es convertida en lluvia.

En cuanto al segundo esquema utilizado, el KF, los procedimientos básicos de operación son similares a los del esquema $\mathrm{G}$, excepto por factores como los siguientes: (a) la inhibición de convección se evalúa en términos de área negativa y no de la profundidad de la cap, (b) no se requiere desestabilización de gran escala para disparar la convección, únicamente energía potencial convectiva disponible (CAPE, en inglés) positiva y (c) las formulaciones de los movimientos descendentes y ascendentes son más sofisticadas, entre otros.

Aunque el modelo MM5v3 es de pronóstico del tiempo (MPT), trabajos como los de Huth et al. (2001) y Huth et al. (2003) sugieren la posibilidad de utilizar los MPT en aplicaciones de modelado climático regional. Por otro lado, Qian et al. (2003) evalúan, con resultados positivos, un método que consiste en la realización de integraciones de corto plazo de un modelo regional y a partir del conjunto de salidas que se obtienen, elaborar o generar predicciones del comportamiento de los patrones climáticos de interés.

\section{Datos y resultados de modelos utilizados}

Los datos y observaciones utilizados en este trabajo estan descritos en detalle en RA1. Para apoyar la evaluación de los resultados, se incorpora el uso de los datos de CRN-073 que tienen una resolución de $0.5^{\circ} \times 0.5^{\circ}$. Además, se emplean datos de cinco estaciones en superficie ubicadas en diferentes puntos de Centroamérica que presentan reportes de precipitación acumulada para el mes en estudio. Estas estaciones son Limón $\left(10^{\circ} \mathrm{N}, 83^{\circ} \mathrm{O}\right)$, San Andrés $\left(12.40^{\circ} \mathrm{N}, 81.40^{\circ} \mathrm{O}\right)$ y Belice $\left(17.50^{\circ} \mathrm{N}, 88.30^{\circ} \mathrm{O}\right)$, en el sector del Caribe y Puntarenas $\left(9.97^{\circ} \mathrm{N}, 84.83^{\circ} \mathrm{O}\right)$ y Managua $\left(12.10^{\circ} \mathrm{N}, 86.20^{\circ} \mathrm{O}\right)$, para el Pacífico (ver Figura 1 de RA1).

Al examinar la capacidad de los dos modelos globales de reproducir el comportamiento de las variables de viento en $925 \mathrm{hPa}$ y precipitación se pudo concluir que el ECHAM4.5 es el que tiene una mejor aproximación a las observaciones (RA1). Sin embargo, como en todo modelo, existen algunas deficiencias en la representación.

Uno de los argumentos principales para esta deducción se encuentra, sin duda, en la mejor habilidad que tiene el ECHAM4.5 de simular el ciclo anual del viento sobre la región de la CCMIA, en comparación con la inadecuada representación que tiene el CCM3.6, especialmente durante el verano boreal. Esto es observable tanto en los promedios espaciales como en la serie de tiempo generada para un área en el Caribe. Además, el ECHAM4.5 muestra las ramificaciones de la corriente en chorro de bajo nivel hacia al norte o al sur, dependiendo de la estación del año, aunque durante el invierno boreal se subestima en 2 m/s o más la magnitud del flujo que se dirige hacia Suramérica. 
En cuanto a la circulación en el Pacífico de la región, el modelo CCM3.6 evidencia carencias importantes en el mes de julio, ya que tiende a representar un mínimo en la intensidad del viento en $925 \mathrm{hPa}$, mientras que en el Reanálisis es destacable el paso de viento desde el Caribe hacia el Pacífico producto de la influencia de la CCMIA durante dicho mes. El ECHAM4.5 parece reproducir con mejores resultados el ciclo anual del viento sobre la mencionada área.

Con respecto a la precipitación, una de las características que más influye en la elección del modelo ECHAM4.5 es la representación del veranillo durante los meses de julio-agosto para la zona del Pacífico. Aunque la serie de tiempo de la Figura 14b de RA1 indica que este modelo sobreestima la cantidad de lluvia, se puede notar que existe una tendencia a la disminución de los valores de esta variable, aunque con un sesgo hacia agosto, y se logra apreciar un comportamiento bimodal.

Durante los episodios de El Niño (La Niña) la magnitud de la CCMIA para los meses del verano boreal que es simulada por el ECHAM4.5 experimenta un aumento (disminución) y esto concuerda con los resultados de las observaciones.

Los patrones de precipitación a gran escala observados para las fases del ENOS parecen coincidir también con los resultados de las simulaciones del ECHAM4.5, ya que se tiene menos (más) lluvia sobre Centroamérica durante los eventos El Niño (La Niña).

\section{Métodos y diseño del experimento}

Los datos para el proceso de reducción de escala usando el modelo regional MM5v3 fueron generados en las facilidades computacionales del Grupo de Prediccion Climática del CIGEFI. Para este trabajo se utilizan las salidas cada seis horas (en el dominio de integración del modelo) para el mes de enero del año 2000 de varios miembros generados en dos dominios: uno madre de $90 \mathrm{~km}$ de resolución (DM) y otro anidado de $30 \mathrm{~km}$ de resolución (DA) (ver Figura 1 en RA1).

Las condiciones iniciales y las condiciones de contorno laterales en el tiempo fueron provistas por el ECHAM4.5 de acuerdo con lo discutido en RA1.

Se escoge el mes de enero del 2000 como el período a simular. La razón principal para esta determinación se debe a que climatológicamente en el primer mes del año la época seca está ampliamente establecida en el Pacífico centroamericano en contraste con la región del Caribe muy húmeda y sometida a empujes polares. Es también importante la presencia de las corrientes en chorro que se producen en el océano Pacífico debido a la presencia de los pasos montañosos de la región bajo el predominio de un campo alisio muy estable.

Con el fin de lograr resultados con mejores resoluciones espaciales, se utilizan dos dominios: uno de 50 x 55 puntos de rejilla separados por una distancia de $90 \mathrm{~km}$ y centrado en $12^{\circ} \mathrm{N}$ y $84^{\circ} \mathrm{O}$ que cubre principalmente Centroamérica, México y el Caribe (dominio madre: DM), y un segundo dominio, anidado en el primero y centrado específicamente en Centroamérica, de 79 x 76 puntos de rejilla separados por una distancia de $30 \mathrm{~km}$ (dominio anidado: DA). La Figura 1 de RA1 muestra la configuración de estos dominios.

Para analizar las salidas del modelo regional y su incertidumbre asociada, se obtiene 
una muestra de doce miembros que consiste en diferentes realizaciones del modelo que inician el mismo día y hora pero que varían en: (a) los datos que proveen las condiciones iniciales y de contorno y (b) los esquemas de parametrización de cúmulos, en donde seis miembros utilizan el esquema $\mathrm{G}$ y los otros seis miembros el esquema KF. Para cada miembro se mantuvieron fijos los esquemas de humedad explícita (hielo simple), de capa límite (MRF), de radiación (radiación-nubes), de temperatura del suelo (capas múltiples de suelos) y no se consideró la presencia de convección poco profunda. Los resultados de estos miembros, generados por la reducción de escala, son promediados para obtener el valor mensual de las variables de viento en $925 \mathrm{hPa}$ y de la precipitación acumulada. Seguidamente, son evaluados mediante la comparación con los patrones climáticos derivados de los conjuntos de datos de observaciones (ver RA1 para una descripción más detallada). Los datos del Reanálisis de NCEP/NCAR y de CMAP son interpolados a las rejillas de 90 km y 30 km de resolución del MM5v3 con el propósito de efectuar el análisis comparativo. Para el caso del viento en $925 \mathrm{hPa}$ se reduce la densidad de puntos de rejilla que se despliegan para poder apreciar más claramente los patrones generados por el MM5v3 y por las observaciones. En todos los casos, los resultados de las primeras 6 horas de integracin no fueron utilizados para evitar desbalances en los campos de las variables meteorolgicas.

La información de estaciones meteorológicas se usa también para evaluar la precipitación generada por el promedio de los miembros del MM5v3. En este caso, se toman los cinco puntos del dominio de $30 \mathrm{~km}$ de resolución que se encuentran más cercanos a cada estación y se promedian para luego compararse con los datos observados.

Finalmente y como un caso particular, se toma el promedio de los dos miembros del MM5v3 que se generaron con datos iniciales del promedio de los cinco miembros de ECHAM4.5 y se compara con el promedio de dos miembros inicializados por iguales datos del modelo global que se ejecutan de manera continua por el mes de enero del 2000. El propósito de este caso es seguir el procedimiento presentado por Qian et al. (2003) y obtener una primera impresión de cuál método (reiniciación o integración continua) podría proveer mejores simulaciones y predicciones estacionales del clima centroamericano.

La capacidad de predicción que tiene el modelo regional se calcula mediante la desviación estándar espacial. Ésta, a pesar de ser una medida estadística sencilla, permite conocer aquellas regiones en las cuales hay más dispersión o variabilidad y por ende, más incertidumbre. También permite determinar aquellas zonas en las que es posible tener una mayor confianza en la simulación obtenida.

Debido a que en este trabajo se generan doce miembros distintos (doce ralizaciones estadísticamente independientes), se utiliza la desviación estándar para una muestra, ya que este número de miembros es pequeño.

El cálculo de este estadístico está dado, de acuerdo con Wilks (1995), de la siguiente manera:

$$
s=\sqrt{\frac{1}{n-1} \sum_{i=1}^{n}\left(x_{i}-\bar{x}\right)^{2}}
$$

donde $s$ es la desviación estándar, $x_{i}$ el elemento $i$ de la muestra, $\bar{x}$ el promedio, y $n$ el número de elementos de la muestra. 


\section{$5 \quad$ Resultados de la reducción de escala con el MM5v3}

Por ser el DM un dominio de una escala espacial grande (ver Figura 1 en RA1), se evalúan aspectos generales del comportamiento del clima para el mes de enero del 2000 y no se pretende encontrar en la simulación una buena representación de aspectos a escala subregional. Aún así, si se comparan los datos provenientes del ECHAM4.5 con la información que se desprende del MM5v3, a simple vista es posible apreciar un mayor nivel de detalle en éste último que no se encuentra en el modelo de circulación general. Para confirmar esta afirmación, a continuación se presentan los análisis del campo de viento en $925 \mathrm{hPa}$ y de precipitación para este primer dominio. Debido a la gran cantidad de puntos que se tienen en el MM5v3, se reduce la densidad de puntos en la representación espacial del viento con el fin de apreciar mejor los patrones de circulación simulados por este modelo.

La Figura 1 muestra el viento en $925 \mathrm{hPa}$ para enero del 2000 de acuerdo con (a) el Reanálisis de NCEP/NCAR interpolado a la rejilla del DM y (b) el promedio de los doce miembros del modelo MM5v3. Nótese que debido a la falta de datos observados en rejillas de altas resoluciones, se emplea el Reanálisis para evaluar el comportamiento de los patrones generales de viento.

Una de las características que se capturan en el proceso de reducción de escala es la región de máximo de viento de bajo nivel que se presenta sobre el mar Caribe, relacionado con la presencia de la CCMIA (Amador et al., 2006). También, existe una ramificación del flujo hacia Suramérica, al este de la cordillera de los Andes. Debido a que la época del año de este experimento corresponde al verano en el Hemisferio Sur, la presencia de este desvío tiene consistencia con las observaciones provenientes del Reanálisis.

De acuerdo con el Reanálisis, existe al sur de la región centroamericana, entre el ecuador y $\operatorname{los} 6^{\circ} \mathrm{N}$, una región en donde la magnitud del viento es relativamente débil. El producto derivado del MM5v3 muestra en buena medida este comportamiento que puede estar muy relacionado con el posicionamiento de la Zona de Convergencia Inter-Tropical (ZCIT) en esa época del año 2000.

Además, en la Figura 1 es posible observar cómo el proceso de reducción de escala arroja resultados que muestran el papel que desempeña la topografía de la región en la canalización del viento a través de los diferentes pasos (por ejemplo, en Tehuantepec y el Golfo de Papagayo) y la habilidad del MM5v3 de discriminar estos procesos de interacción. No es posible identificar este comportamiento en la representación de un modelo global como el ECHAM4.5 ni en el Reanálisis, puesto que la resolución que poseen no es lo suficientemente fina para ello.

La medida de dispersión o incertidumbre en los resultados del MM5v3 para la variable de magnitud de viento en $925 \mathrm{hPa}$ se presenta en la Figura 2. De acuerdo con esta figura, donde se observa una mayor desviación estándar (entre 3 y $4 \mathrm{~m} / \mathrm{s}$ ) es sobre el Golfo de Panamá y desde ahí hacia el suroeste hasta los $3^{\circ} \mathrm{N}$, aproximadamente. Nótese que esta es una zona con deficiencias importantes de datos derivados de observaciones, lo que repercute en los valores iniciales y por ende en los resultados de la simulación. Otras zonas importantes, con valores de desviación entre los 2 y $3 \mathrm{~m} / \mathrm{s}$, son: (a) el este de la cordillera de los Andes que, como se ha discutido, está ligada al desvío en el campo de viento producto de los contrastes térmicos entre tierra y mar; (b) el Golfo de Tehuantepec, 


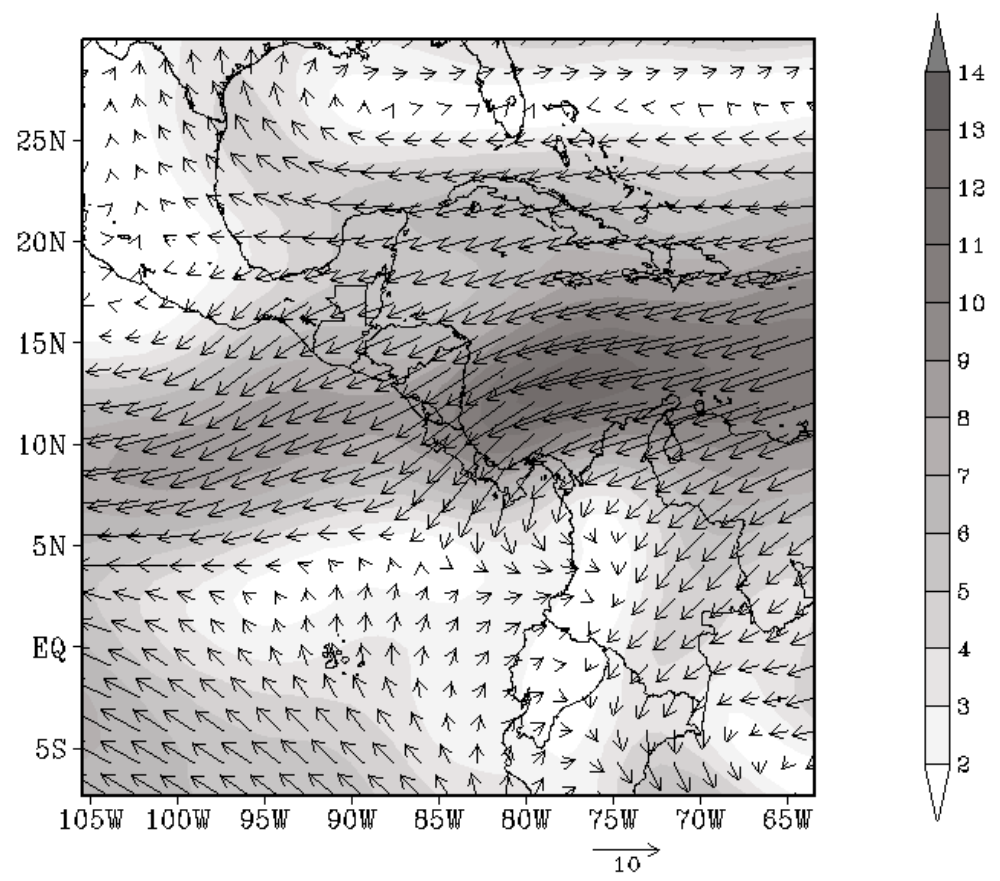

(a)

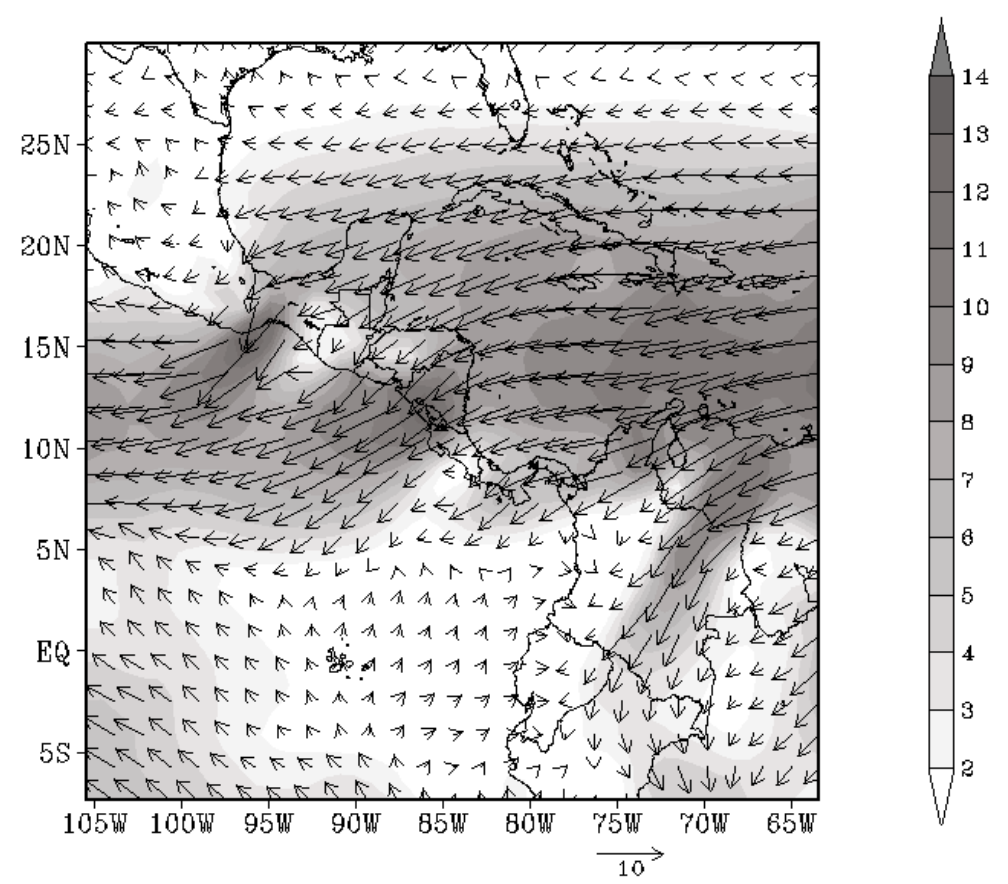

(b)

Figura 1: Viento promedio en $925 \mathrm{hPa}(\mathrm{m} / \mathrm{s})$ para enero 2000 para (a) el Reanálisis NCEP/NCAR y (b) el promedio de los miembros del MM5v3 (DM). 
lo cual indica una incertidumbre en cuanto a la magnitud del viento que se canaliza por el paso de Chivela y (c) la región desde las Antillas Mayores hasta el sur de la península de Florida.

Cabe resaltar que entre las áreas donde la desviación estándar para la simulación de enero del 2000 no supera los $2 \mathrm{~m} / \mathrm{s}$, tenemos el mar Caribe, donde los miembros parecen mostrar un consenso en cuanto a la presencia de una corriente en chorro o un máximo en la intensidad del viento.

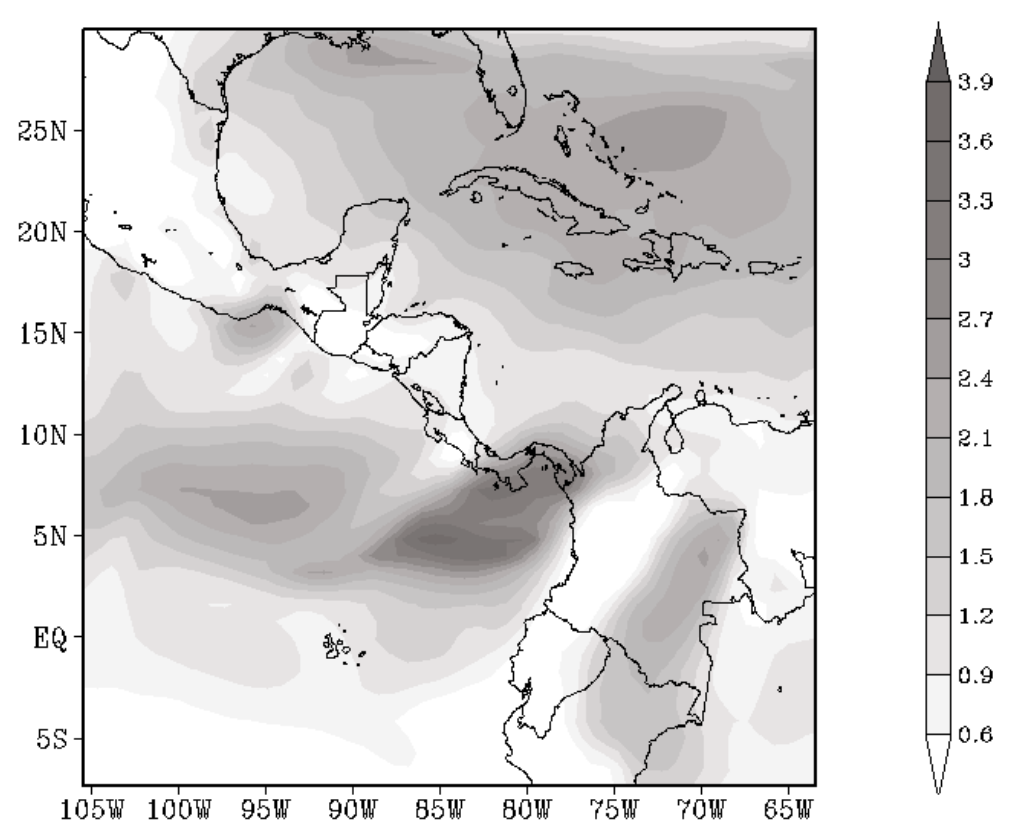

Figura 2: MM5v3: Desviación estándar de la magnitud del viento en $925 \mathrm{hPa}(\mathrm{m} / \mathrm{s})$ para enero 2000 (DM).

La Figura 3 muestra la precipitación para enero 2000. La comparación de las estimaciones de CMAP (Figura 3a) con el promedio de los doce miembros del MM5v3 (Figura 3b), indica que el modelo regional simula en buen grado la posición de la ZCIT al sur de Centroamérica, entre el ecuador y $\operatorname{los} 6^{\circ} \mathrm{N}$, aproximadamente. Esta condición es típica de la estación seca que predomina en los sectores cercanos a la costa Pacífica de la región durante el mes de enero. Precisamente, el resultado de la reducción de escala presenta un área de menos precipitaciones en el mencionado sector. Contrario a esta situación, tanto los datos de CMAP como los del MM5v3 reflejan una situación de condiciones más lluviosas a lo largo del caribe centroamericano que posiblemente pueden ser producto de la influencia de la CCMIA y de las interacciones entre la topografía y los empujes polares propios de la época. También son destacables algunos problemas en los resultados de la simulación. Por ejemplo, en el sur de México existe una franja lluviosa con orientación 
norte-sur que no guarda consistencia con el patrón estimado por CMAP, sin embargo es difícil determinar la causa de esta condición.

Al observarse la desviación estándar de la precipitación desplegada en la Figura 4, se nota una alta dispersión (de hasta $500 \mathrm{~mm}$ ) al sur de Panamá, también frente a las costas caribeñas de este país y de Costa Rica y sobre la citada franja norte-sur que se ubica al sur de México. Al igual que con lo sucedido con la desviación estándar del campo de viento, la falta de observaciones principalmente en el sur de Panamá parece afectar el desempeño del MM5v3 sobre esta zona.

Como se aprecia en la Figura 1 de RA1, el dominio de 30 km de resolución está definido sobre la región centroamericana. Esta es la reducción de escala de mayor resolución (unos $0.3^{\circ} \times 0.3^{\circ}$ ) establecida para los efectos del presente trabajo y se presta atención particular a las características regionales que el MM5v3 puede o no simular. Al igual que en el caso del dominio de $90 \mathrm{~km}$ de resolución, se utilizan menos puntos de rejilla para representar el campo de viento en $925 \mathrm{hPa}$, con el fin de hacer más clara la visualización de la información generada por el modelo regional.

El análisis de campo de viento en $925 \mathrm{hPa}$ presentado en la Figura 5 demuestra la capacidad del MM5v3 para representar el efecto de canalización del viento que viene del Caribe hacia el Pacífico a través de los pasos existentes en el sistema montañoso, como lo son el de Panamá y el de los Golfos de Fonseca y de Papagayo, cerca del Lago de Nicaragua. Las zonas en tierra donde se producen mínimos relativos en la magnitud del viento están relacionadas con la interacción del flujo con la topografía. Al comparar nuevamente con los datos del Reanálisis de NCEP/NCAR, es evidente que el modelo regional está capturando características climáticas regionales que no se pueden apreciar cuando se trabajan con resoluciones menos finas.

La magnitud del máximo de viento simulado sobre el mar Caribe (aproximadamente $10 \mathrm{~m} / \mathrm{s}$ ), en la región de la CCMIA, parece ser menor que la magnitud observada en el Reanálisis (unos $12 \mathrm{~m} / \mathrm{s}$ a $13 \mathrm{~m} / \mathrm{s}$, ver Figura 5), aunque el patrón del MM5v3 sobre dicha región coincide con el de las observaciones.

La desviación estándar de la Figura 6 evidencia valores superiores a los $3 \mathrm{~m} / \mathrm{s}$ al sur de Panamá. Considerando que el promedio de los doce miembros presenta, para enero del 2000, un valor de viento en $925 \mathrm{hPa}$ que ronda los $5 \mathrm{~m} / \mathrm{s}$, se puede inferir que en el modelo regional existe una mayor incertidumbre en cuanto a la ubicación de la ZCIT, ya que ésta tiene una posición que depende del comportamiento del flujo alisio.

Para la mayor parte del istmo centroamericano, los miembros de las simulaciones del MM5v3 apuntan a una menor dispersión en los valores de viento. La desviación estándar en esa región no supera los $2 \mathrm{~m} / \mathrm{s}$. Similar situación ocurre sobre el mar Caribe occidental.

La Figura 7 presenta la precipitación acumulada para enero del 2000 tanto para las observaciones (CRN-073 y CMAP) como para el promedio de los miembros del MM5v3 con resolución de $30 \mathrm{~km}$. Es posible notar en el resultado de la simulación un importante contraste entre el patrón de precipitación entre el Caribe y el Pacífico de Centroamérica, consistente con lo que muestran las observaciones. En la primera región se observa para el MM5v3 un máximo relativo de lluvia frente a las costas orientales de Costa Rica y el sureste de Nicaragua, producto de la convergencia generada por la CCMIA. También, es importante el efecto del forzamiento orográfico en la generación de precipitaciones al este 

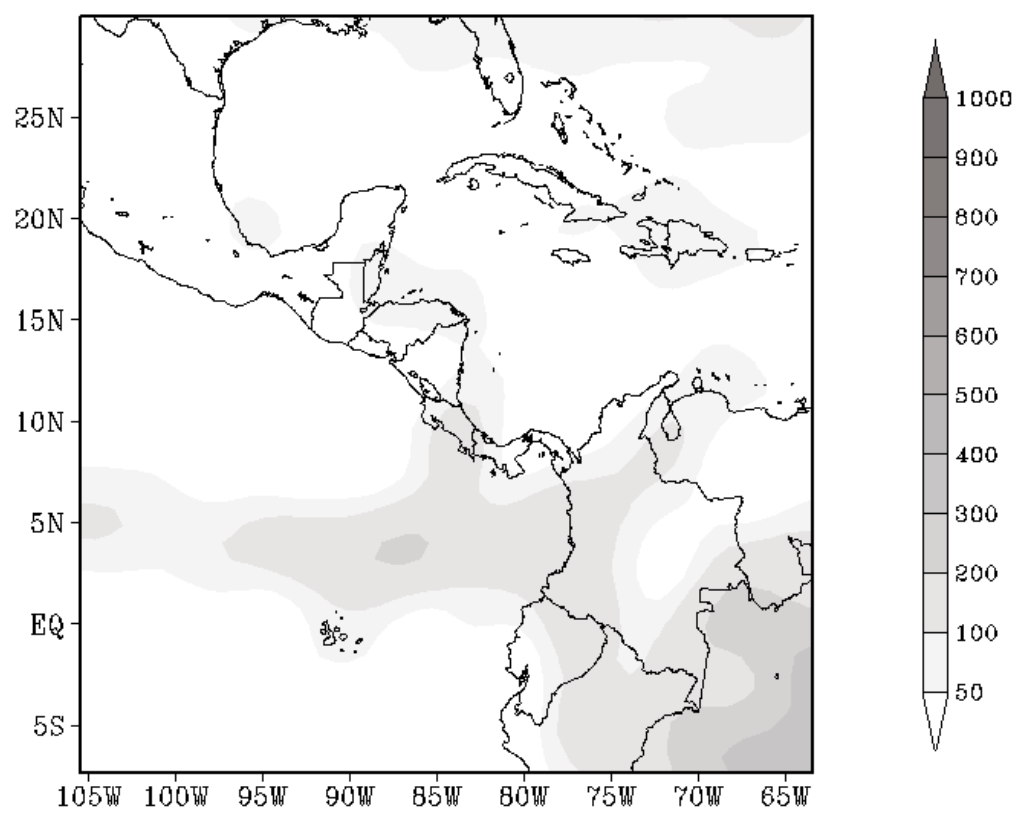

(a)
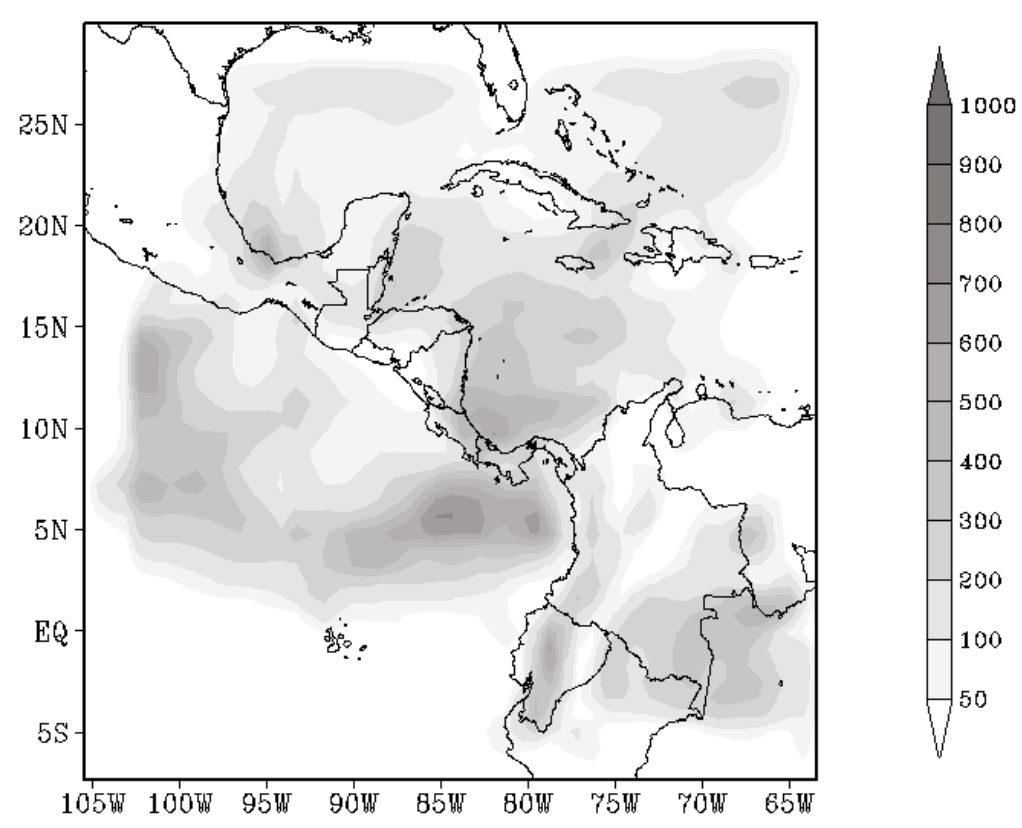

(b)

Figura 3: Precipitación (mm) para enero 2000 para (a) el CMAP y (b) el promedio de los doce miembros del MM5v3 (DM). 


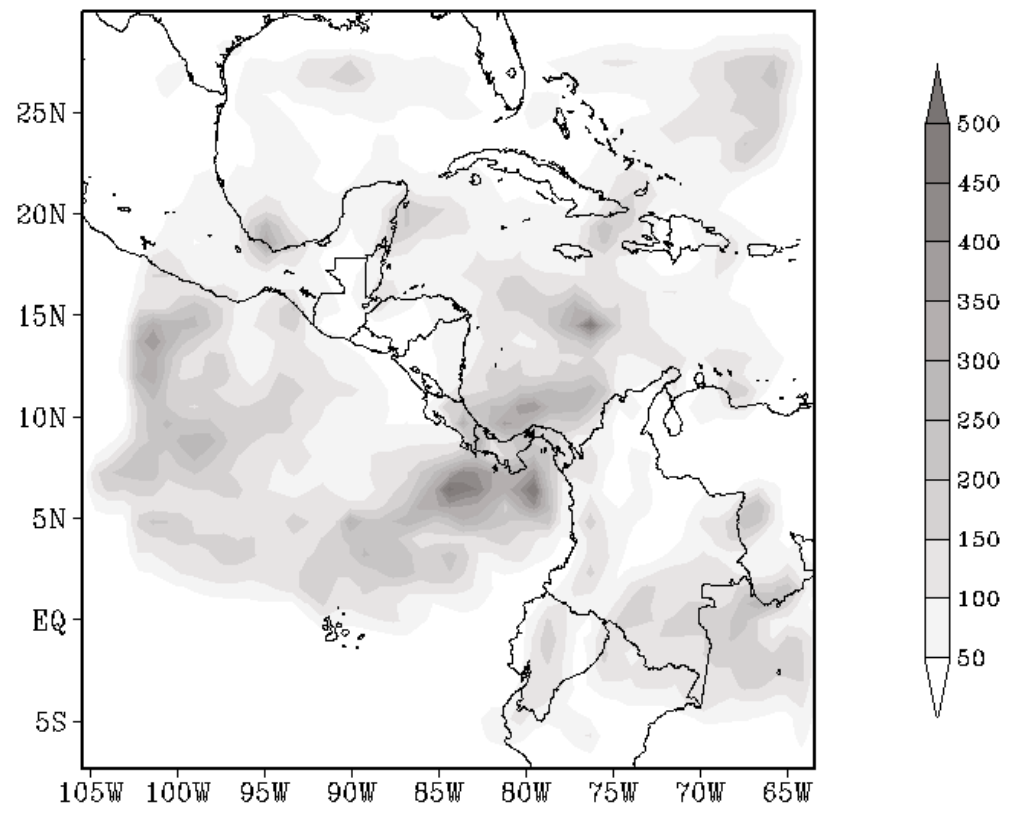

Figura 4: M5v3: Desviación estándar de precipitación (mm) para enero 2000 (DM). 

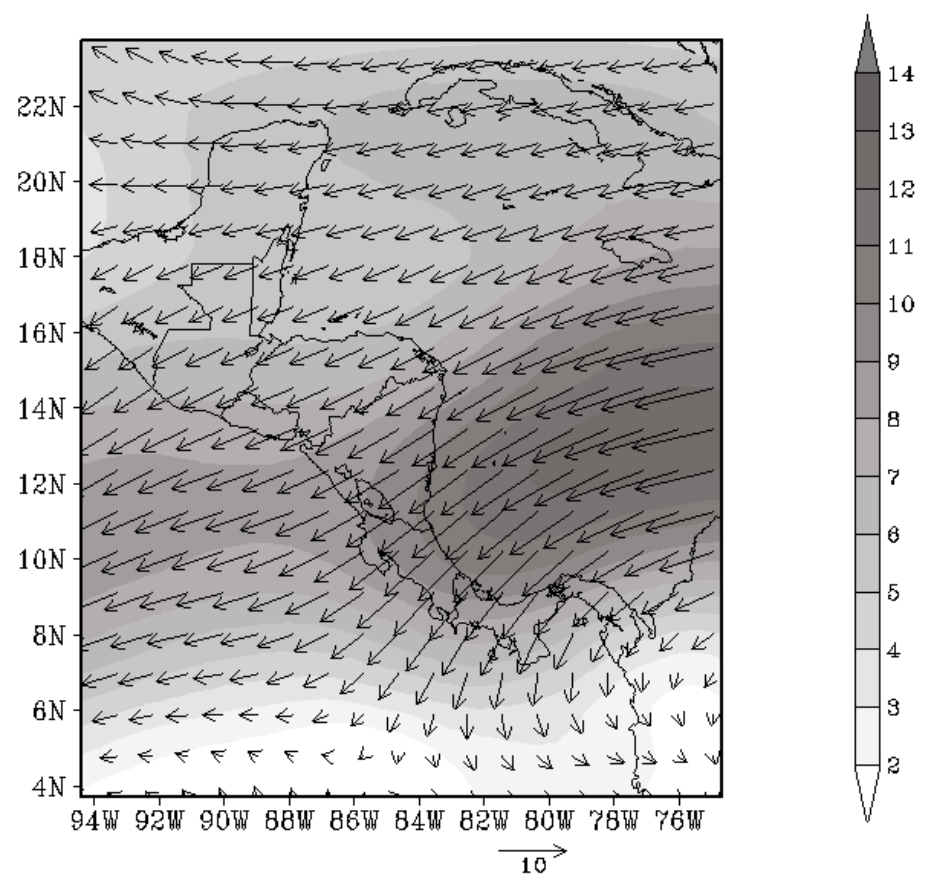

(a)

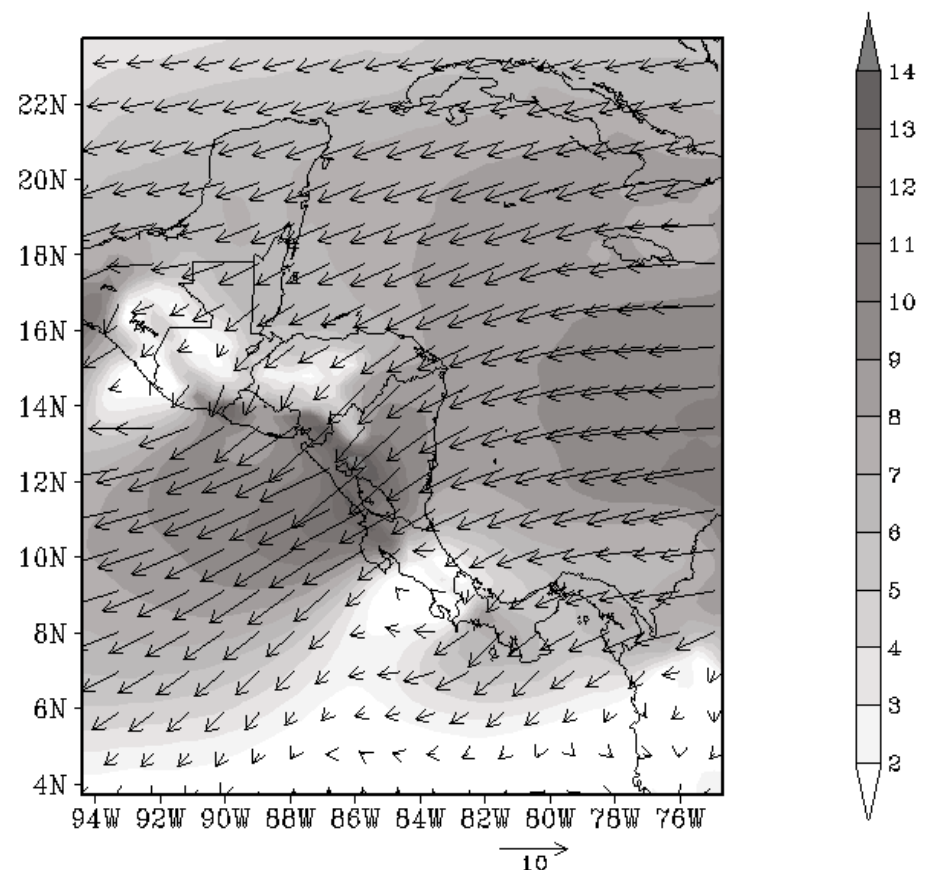

(b)

Figura 5: Viento promedio en $925 \mathrm{hPa}(\mathrm{m} / \mathrm{s})$ para enero 2000 para (a) el Reanálisis NCEP/NCAR y (b) el promedio de los miembros del MM5v3 (DA). 


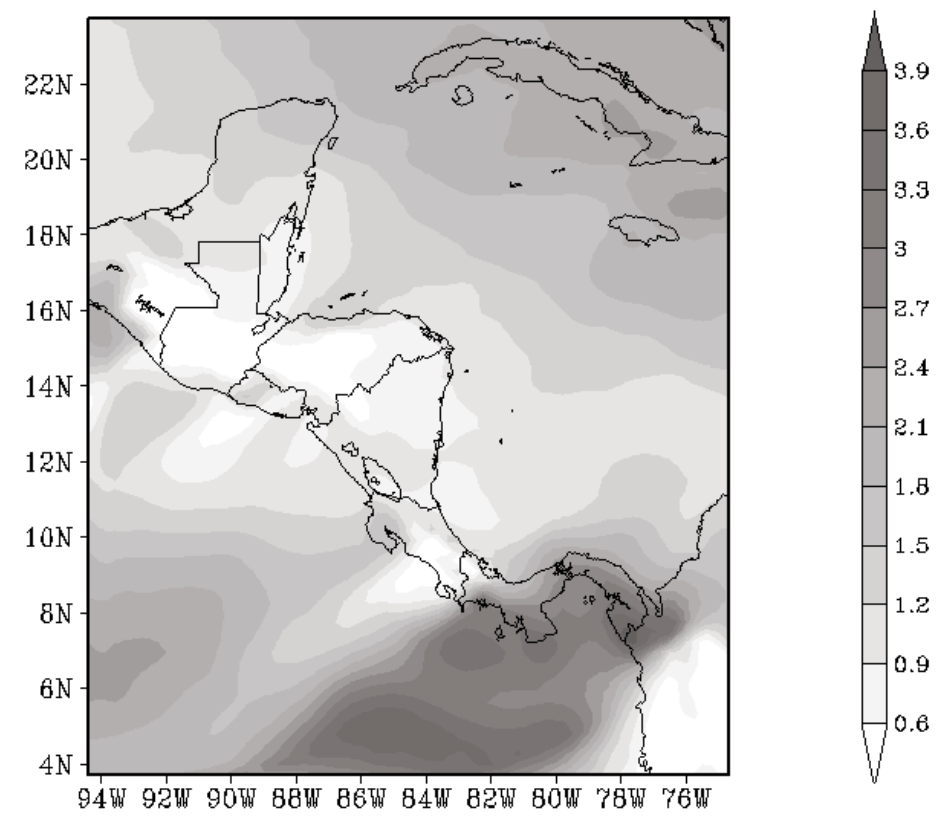

Figura 6: MM5v3: Desviación estándar de la magnitud del viento en $925 \mathrm{hPa}(\mathrm{m} / \mathrm{s})$ para enero 2000 (DA). 
de los sistemas montañosos, que en parte pueden ser producidas por los empujes de aire frío desde el norte que son característicos de la época invernal en el Hemisferio Norte. En la segunda región, por el contrario, se produce un descenso del flujo y, por calentamiento adiabático, se inhibe la formación de nubes, dando lugar a una zona de escasas lluvias. Es de resaltar, sin embargo, que la cantidad de lluvia simulada en el modelo regional sobrepasa en casi toda la región de Centroamérica los valores que se muestran en las observaciones, lo cual es un indicativo de que existen en los esquemas de parametrización utilizados ciertas dificultades para generar cantidades más realistas de precipitación.

La ZCIT en el modelo regional se ubica al sur de Centroamérica, aunque parece estar más desplazada hacia el norte en comparación con lo mostrado por las observaciones. Como se explicó anteriormente, esta situación puede deberse a las incertidumbres mostradas en el patrón de viento de bajo nivel sobre el sur de Panamá y posiblemente a las asociadas con el campo de humedad.

La Figura 8 refuerza la afirmación del párrafo anterior al mostrar los altos valores de desviación estándar sobre la zona en mención. En general, en el Pacífico centroamericano se muestra un consenso por parte de los miembros en cuanto al reconocimiento de condiciones secas.

El Cuadro 1 muestra la comparación entre los datos de estaciones meteorológicas y el promedio de los cinco puntos de rejilla más cercanos a cada estación del dominio de 30 km de resolución del MM5v3. La información que se deriva de dicha tabla confirma el hecho de que el modelo tiende a producir más lluvia de la observada, pues en cuatro de los cinco promedios de puntos cercanos a las estaciones de San Andrés, Belice, Puntarenas y Managua se da este comportamiento. A pesar de esta situación, se puede notar que el MM5v3 produce más precipitación en la región del Caribe (337 mm, en promedio) que en el Pacífico (45 mm, en promedio, lo cual concuerda con los datos de las estaciones (226 $\mathrm{mm}$, en promedio para las del Caribe y $2 \mathrm{~mm}$, en promedio para las del Pacífico) y ratifica el análisis derivado de la Figura 7.

En cuanto al desempeño de los dos esquemas de parametrización de cúmulos utilizados en el experimento, la Figura 9 es una comparación entre los resultados de los seis miembros elaborados con el esquema de Grell (MM5G) y los seis miembros configurados con el esquema de Kain-Fritsch (MM5K). Se puede notar que en MM5G la zona de lluvias asociadas con la ZCIT se encuentra más al sur de lo que muestra MM5K y más acorde con los resultados observados. En cuanto al máximo de lluvias al este de Costa Rica, se aprecia un corrimiento más al este por parte de MM5K de esta característica con respecto a MM5G. El patrón que se presenta en las observaciones (Figuras 7a y 7b) parecen coincidir de mejor manera con el patrón simulado por MM5G.

Otra particularidad digna de mención es que las diferencias entre MM5G y MM5K (Figura 9c) demuestran que el primero tiende a producir más lluvia a lo largo de la vertiente caribeña que el segundo, mientras que el comportamiento inverso se da en la vertiente pacífica. En MM5G se nota con mayor claridad el patrón seco del Pacífico.

De fundamental interés, no solo desde el punto de vista computacional, sino físico es la comparación de resultados para los experimentos reinicializados y de integración contínua en el tiempo.

La Figura 10 presenta la comparación para el campo de viento en $925 \mathrm{hPa}$ entre la 

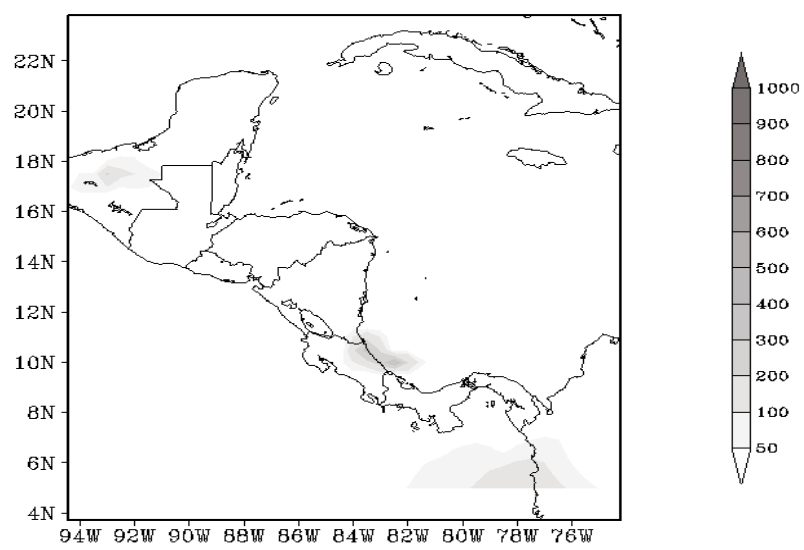

(a)
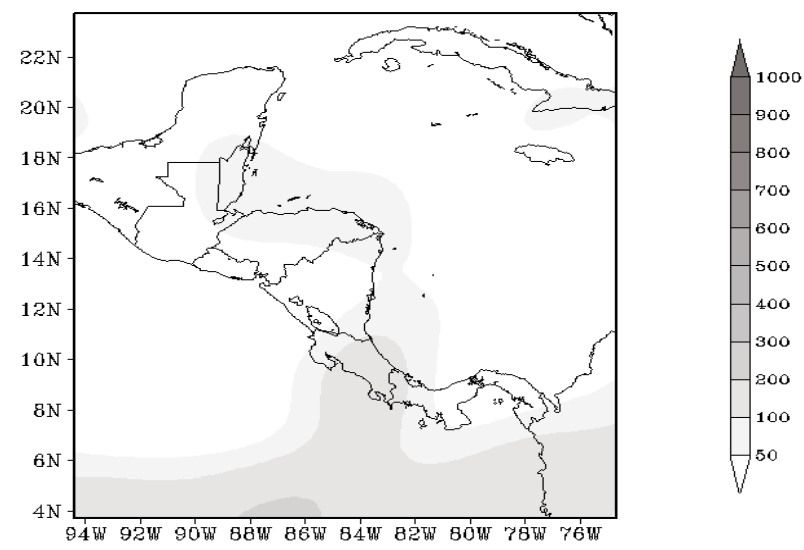

(b)
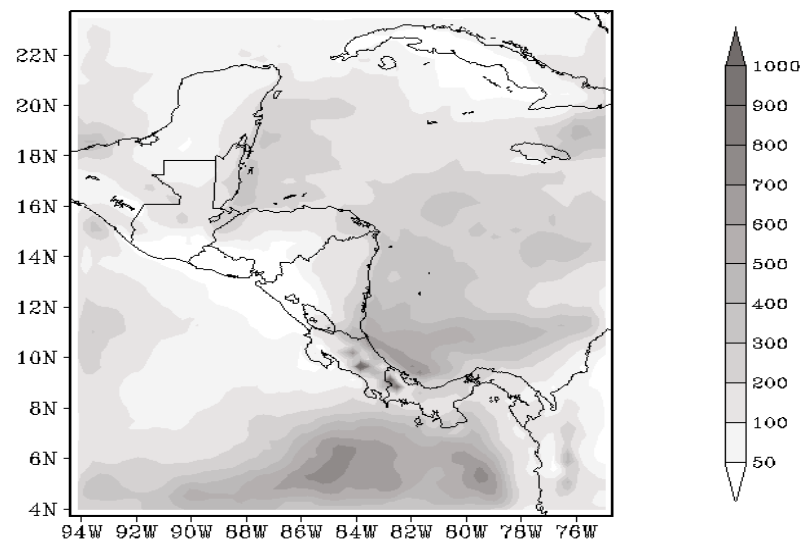

(c)

Figura 7: Precipitación (mm) para enero 2000 para (a) el CRN-073, (b) el CMAP y (c) el promedio de los miembros del MM5v3 (DA). 


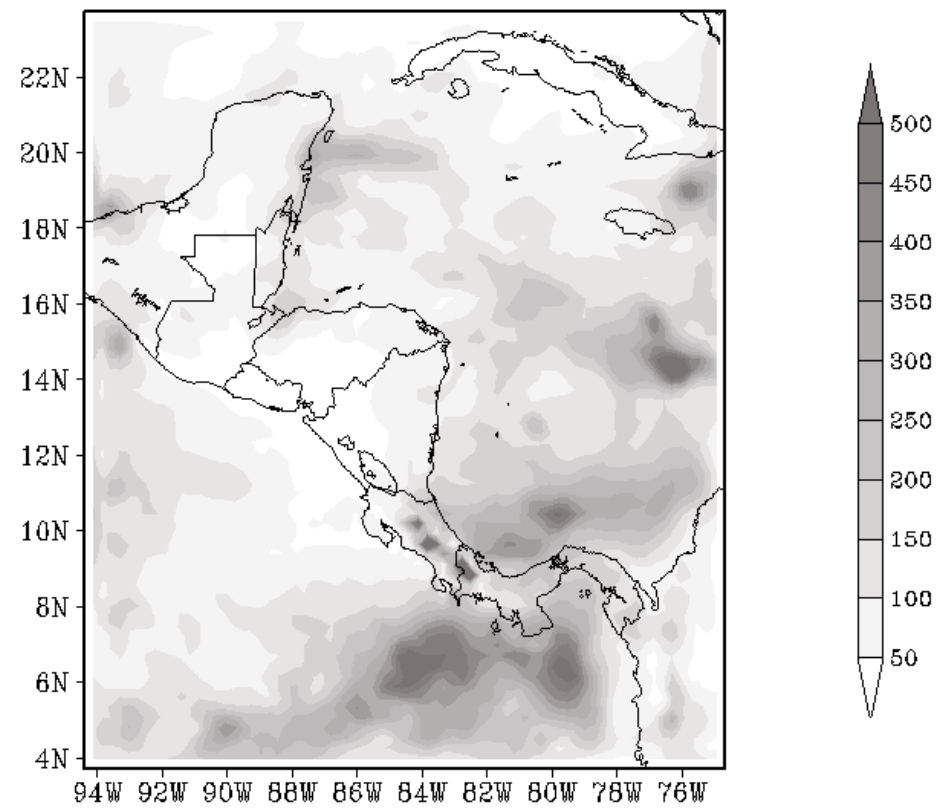

Figura 8: MM5v3: Desviación estándar de la precipitación (mm) para enero 2000 (DA).

\begin{tabular}{|c|c|c|}
\hline \multicolumn{3}{|c|}{ Precipitación Caribe (mm) } \\
\hline Estación & $\begin{array}{c}\text { Observación } \\
\text { BD1 }\end{array}$ & $\begin{array}{c}\text { MM5v3 } \\
\text { BD2 }\end{array}$ \\
\hline Limón & 486 & 406 \\
\hline San Andrés & 90 & 333 \\
\hline Belice & 103 & 271 \\
\hline Promedio & 226 & 337 \\
\hline \multicolumn{3}{|c|}{$\overline{\text { Precipitación Pacífico (mm) }}$} \\
\hline Estación & $\begin{array}{c}\text { Observación } \\
\text { BD1 }\end{array}$ & $\begin{array}{l}\text { MM5v3 } \\
\text { BD2 }\end{array}$ \\
\hline Puntarenas & 1 & 58 \\
\hline Managua & 3 & 32 \\
\hline Promedio & 2 & 45 \\
\hline
\end{tabular}

Tabla 1: Precipitación observada en cinco estaciones meteorológicas (tres en el Caribe, dos en el Pacífico) y la simulada por el modelo MM5v3 tomando en consideración los cinco puntos de rejilla más cercanos a cada estación. 
simulación reiniciada del modelo regional (MM5R) y la simulación continua (MM5C) en el dominio de $30 \mathrm{~km}$ de resolución (DA). Se utilizan los datos interpolados del Reanálisis de NCEP/NCAR para efectuar esta comparación. En las Figuras 10a y 10b se puede apreciar que ambas simulaciones subestiman entre $1 \mathrm{~m} / \mathrm{s}$ y $3 \mathrm{~m} / \mathrm{s}$ la intensidad del viento en la región de la CCMIA. Sin embargo, la Figura 10c muestra que en dicha región MM5C es la que presenta la mayor subestimación. En la región de las corrientes en chorro de los Golfos de Papagayo y Fonseca, las magnitudes del viento para MM5R y MM5C son mayores con respecto al Reanálisis (de hasta $4 \mathrm{~m} / \mathrm{s}$ ), mientras que sobre las cordilleras de Guatemala, Honduras y Costa Rica existen amplias zonas en las cuales estas dos simulaciones presentan valores muy inferiores a las observaciones (de $5 \mathrm{~m} / \mathrm{s}$ o más). Este comportamiento puede ser resultado de que el Reanálisis no es capaz de representar características climáticas regionales debidas a las interacciones del flujo con zonas montañosas principalmente de Guatemala, Honduras y Costa Rica y el efecto de canalización a través de los pasos topográficos, por lo cual se aprecian estas importantes diferencias. En términos generales, MM5R produce magnitudes de viento superiores a MM5C, exceptuando la región de la ZCIT ubicada alrededor de los $5^{\circ} \mathrm{N}$.

En cuanto a la precipitación, la Figura 11 muestra en general una sobreestimación por parte de ambas simulaciones con respecto a las estimaciones de CMAP (Figuras 11a y 11b). Donde se presenta menos diferencia es en el Pacífico centroamericano, ya que el modelo simula condiciones secas en esta región, en correspondencia con las condiciones observadas. En cuanto al sector caribeño, ambas simulaciones capturan el patrón, aunque no la cantidad, de máxima lluvia frente a las costas de Costa Rica y Nicaragua, sin embargo, la Figura 11c evidencia que MM5C genera más lluvia que MM5R, por lo que ésta última se acerca un poco más a CMAP. Por debajo de los $6^{\circ} \mathrm{N}$ se puede ver que MM5R tiende a producir más lluvia que MM5C.

A la luz de los resultados obtenidos para este caso particular, es difícil establecer una conclusión sobre cuál estrategia de modelado es más efectiva para simulaciones o predicciones estacionales. No obstante, parece que la aproximación mediante reinicios del MM5v3 muestra menores errores en comparación con la simulación continua, especialmente en el caso de la región de la CCMIA, en donde los valores de la magnitud del viento se acercan más a los datos del Reanálisis de NCEP/NCAR y en cuanto a la cantidad de precipitación simulada para la vertiente del Caribe de Costa Rica y Nicaragua. Un resultado similar se obtuvo para las simulaciones reiniciadas versus las continuas en el trabajo de Qian et al. (2003), sin embargo, es necesario indicar que en este último trabajo las simulaciones fueron por períodos de hasta cinco meses, en contraste con un período de un mes para el presente trabajo.

\section{Conclusiones}

Las simulaciones con el MM5v3 consistieron en un conjunto de treinta y seis realizaciones numéricas sucesivas o reiniciadas (de diez días cada una, aproximadamente) que dieron origen a doce miembros estadísticamente independientes. Los datos iniciales provinieron de cinco miembros del ECHAM4.5 (ver RA1 para más detalles) más el promedio de los 

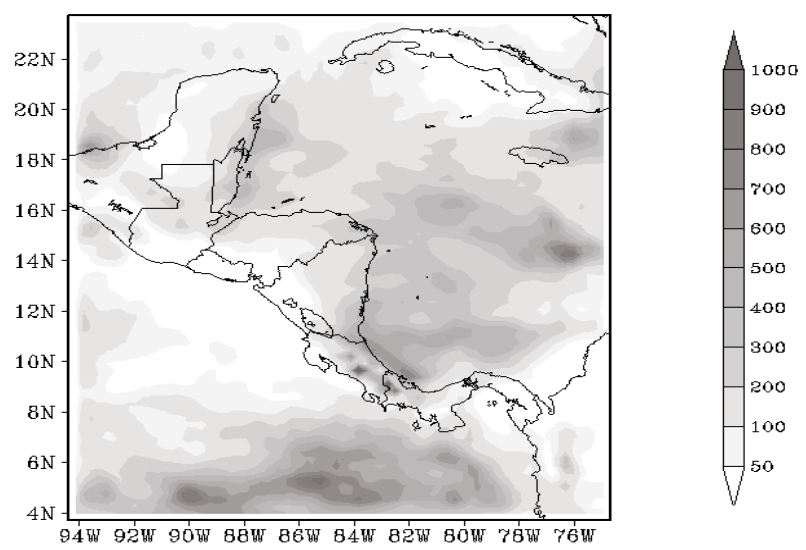

(a)
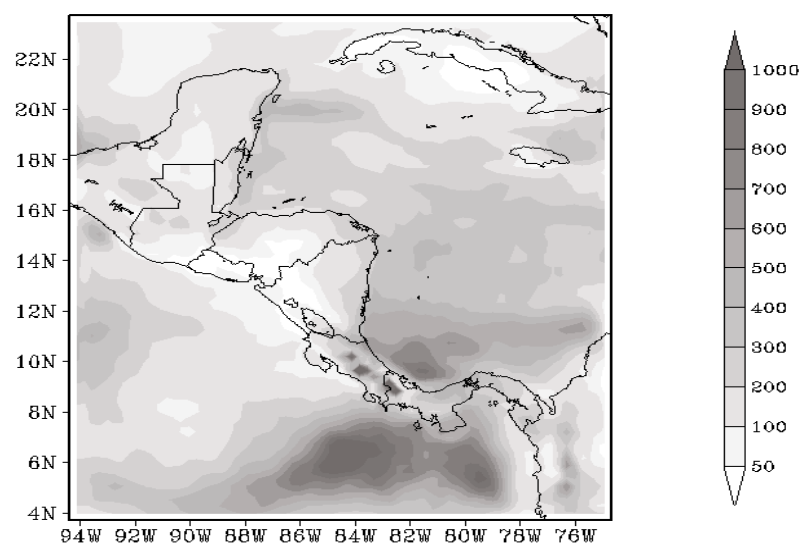

(b)

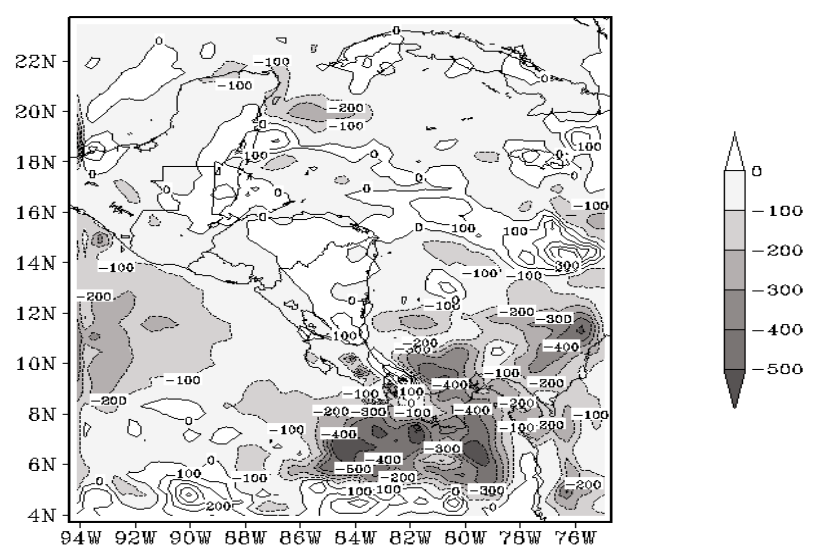

(c)

Figura 9: Precipitación (mm) para enero 2000 para (a) MM5G, (b) MM5K y (c) MM5G menos MM5K (DA). 


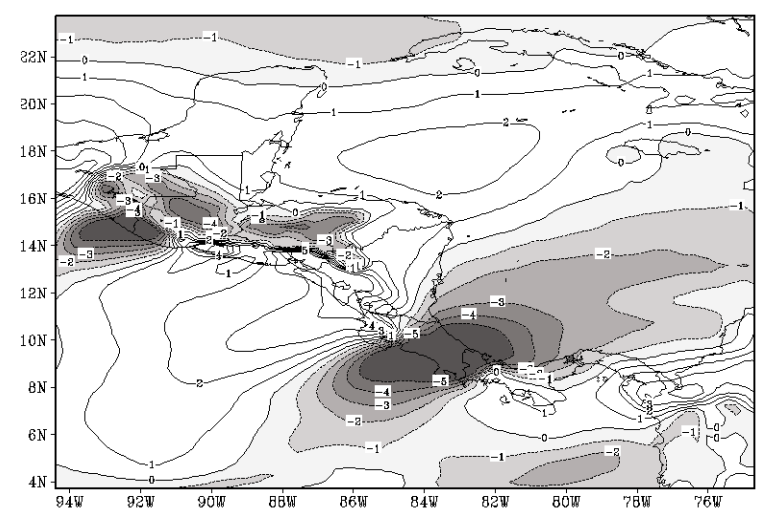

(a)

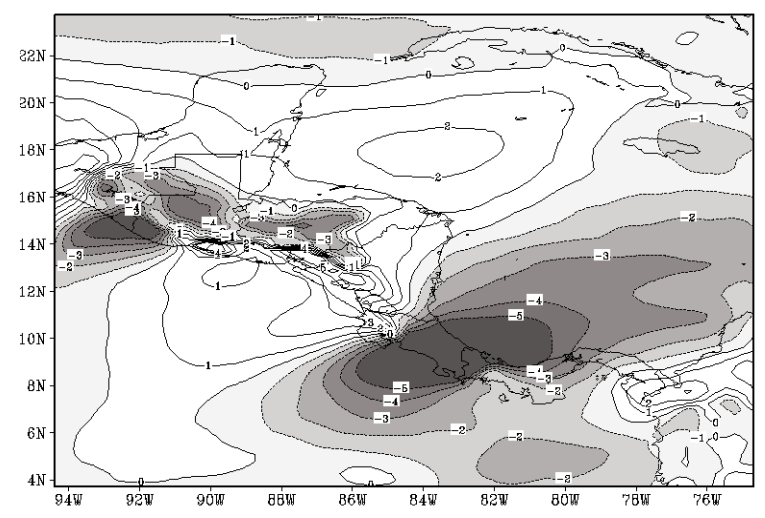

(b)

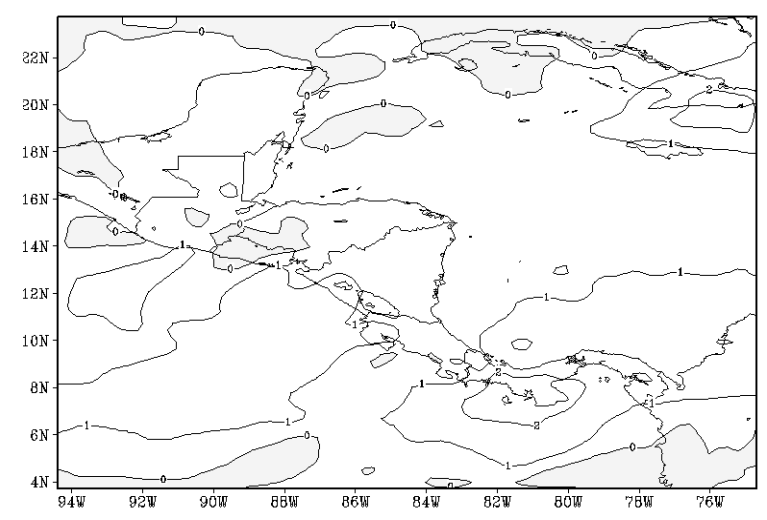

(c)

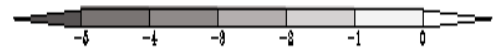

Figura 10: Diferencia del viento promedio en $925 \mathrm{hPa}(\mathrm{m} / \mathrm{s})$ para enero 2000 para (a) MM5R menos el Reanálisis NCEP/NCAR, (b) MM5C menos el Reanálisis de NCEP/NCAR y (c) MM5R menos MM5C (DA). 


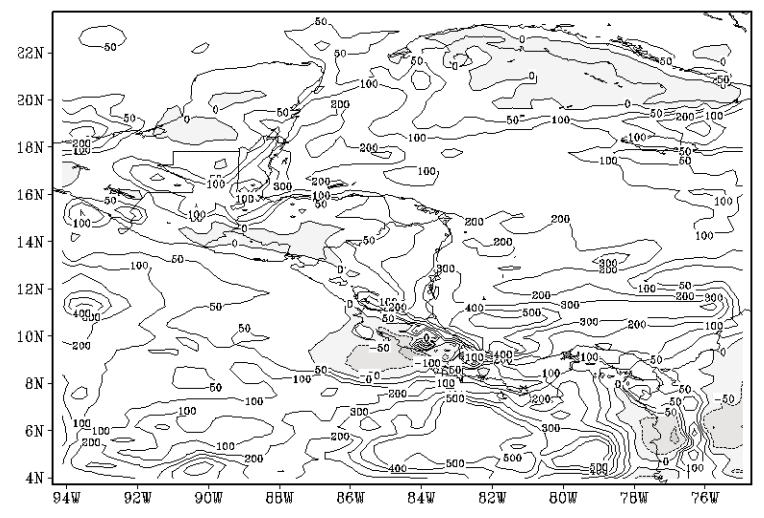

(a)

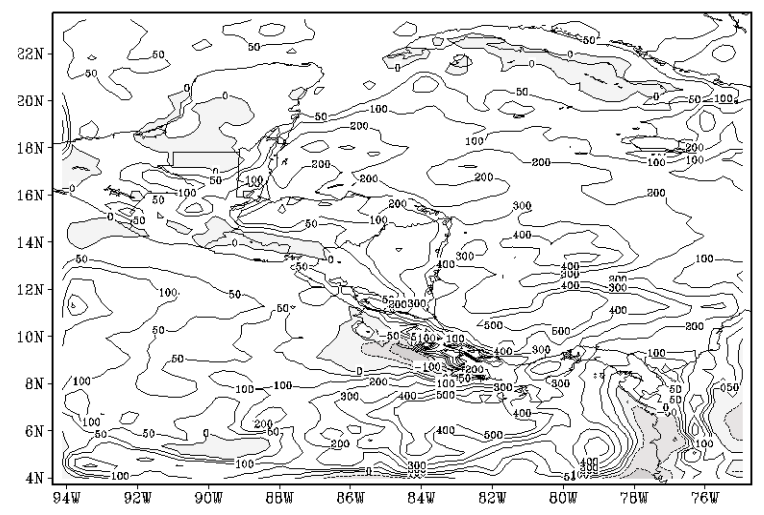

(b)

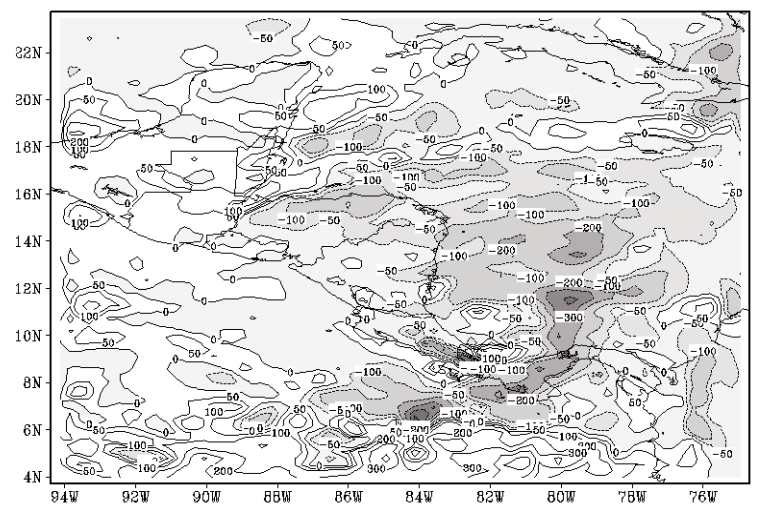

(c)

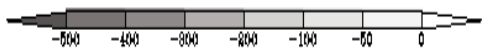

Figura 11: Ídem para Figura 10 excepto para precipitación (mm) para (a) MM5R menos el CMAP, (b) MM5C menos el CMAP y (c) MM5R menos MM5C (DA). 
mismos (en total, seis miembros del modelo global). Asimismo, se utilizaron dos distintas configuraciones físicas del modelo regional que se diferenciaban entre sí por el esquema de parametrización de cúmulos (Grell por un lado y Kain-Fritsch por el otro).

El análisis del promedio de los doce miembros del MM5v3 para el viento en $925 \mathrm{hPa}$ y la precipitación mostró que este modelo logra capturar detalles regionales que no se distinguen en el Reanálisis de NCEP/NCAR ni en el ECHAM4.5, como lo son la canalización del viento a través de los pasos topográficos, que da origen a las corrientes en chorro en el Pacífico, y las interacciones del flujo con los sistemas montañosos principalmente de Guatemala, Honduras y Costa Rica. La zona de máximo viento en $925 \mathrm{hPa}$ en el Caribe fue representada razonablemente bien por el MM5v3, igualmente la ramificación de la CCMIA hacia Suramérica producto del calentamiento diferencial entre la tierra y el océano durante el verano del Hemisferio Sur.

En cuanto a los patrones de lluvia para el mes en estudio, es importante hacer notar que el MM5v3 fue capaz de detectar las diferencias entre la vertiente del Caribe (más lluviosa, especialmente frente a las costas de Costa Rica y Nicaragua) y la vertiente del Pacífico (con una marcada época seca), aunque la comparación con datos observados (estaciones puntuales y análisis espacial) indicó que existe una sobreestimación generalizada de esta variable. Por ejemplo, el promedio de tres estaciones en el Caribe para enero del año 2000 registra un total de $226 \mathrm{~mm}$ de lluvia, mientras que el MM5v3 obtiene en promedio $337 \mathrm{~mm}$. Del mismo modo, al promediar en el Pacífico dos estaciones, la cantidad de lluvia es de 2 mm y en el MM5v3 es de $45 \mathrm{~mm}$. La comparación entre los esquemas de parametrización de cúmulos evidenció que el esquema de Kain-Fritsch tiende a producir mayor cantidad de lluvia que el esquema de Grell, con excepción de algunas zonas continentales de la vertiente caribeña.

La desviación estándar tanto de las muestras de viento como de precipitación indicó que existe una mayor dispersión o incertidumbre espacial al sur de Panamá. Esta situación puede ser debida en parte a carencias en observaciones sobre esta zona, lo cual afecta las condiciones iniciales de los MCG y, como resultado, las que se le proveen al MM5v3.

Con el fin de examinar las ventajas y desventajas de efectuar un conjunto de simulaciones sucesivas o reiniciadas (MM5R) y uno de si-mulaciones continuas (MM5C), se procedió a trabajar un caso particular en el cual los experimentos se inicializaron con el promedio de los cinco miembros disponibles del ECHAM4.5 y utilizaron también dos esquemas de parametrización de cúmulos (Grell y Kain-Fritsch). Los resultados iniciales, que son consistentes con el trabajo de Qian et al. (2003), parecen indicar que MM5R provee una mejor representación de la magnitud del viento en $925 \mathrm{hPa}$ sobre la región de la CCMIA. También se pudo constatar que, en términos generales, MM5C tiende a sobreestimar más la lluvia que MM5R, especialmente en la zona del Caribe de Centroamérica. Es necesario corroborar estos resultados con muestras mayores (mayor número de miembros) y períodos más extensos en el ámbito de la predicción estacional.

Respecto a la reducción de escala dinámica, es importante generar un mayor número de miembros que los empleados en el presente trabajo, ya que esto permitiría tener una muestra más grande con la cual efectuar análisis estadísticos más robustos.

Es importante poder validar los resultados del modelo regional a diferentes niveles en la vertical, no solamente en $925 \mathrm{hPa}$, mediante el empleo de datos de radiosondeos. 
Esto permitiría observar si, por ejemplo, se simula correctamente la estructura vertical de la corriente en chorro de bajo nivel de los Mares Intra-Americanos (CCMIA) y otros elementos del clima conocidos en la región como la estructura media de la atmósfera tropical en estas latitudes.

El modelo MM5v3 se evaluó con dos esquemas de parametrización de cúmulos y se dejaron las demás opciones físicas fijas. Esto hizo factible conocer un poco más en detalle las ventajas y desventajas de uno u otro esquema. Es posible con esta información plantear ajustes, ya sea empíricos o estadísticos, que permitan obtener valores de la precipitación más consistentes con los observados. También, se debe estudiar el desempeño de estos esquemas sobre zonas continentales y océanicas. Adicionalmente, se recomienda aplicar este procedimiento para determinar el efecto que tiene el uso de diferentes esquemas de radiación, capa límite o humedad, por mencionar algunos ejemplos, en los resultados de las simulaciones.

Un punto no considerado en el presente trabajo fue el uso del suelo en el MM5v3. La representación de esta característica parece no ser la más apropiada para la región, por lo que se recomienda utilizar una matriz con información en la frontera inferior más realista acorde con la estación del año que se desea estudiar, ya que una inadecuada representación puede incidir en aspectos como el albedo y los flujos superficiales de energía, humedad y momento, lo que implica balances y procesos energéticos físicamente poco consistentes con la realidad observada.

Dado que el MM5v3 es un modelo paralelizable, el uso de un clúster de alto rendimiento para la realización de procesos de reducción de escala dinámica sin duda constituirá un avance importante, lo cual derivará en simulaciones o predicciones climáticas regionales de alta resolución espacial y a diferentes escalas temporales en tiempos de ejecución razonables.

\section{$7 \quad$ Agradecimientos}

Este trabajo forma parte de la Tesis de Maestría "El Problema y Aplicaciones de la Reducción de Escala Dinámica para la Predicción Climática Estacional en Centroamérica" defendida exitosamente por el primero de los autores (ERR) en octubre del 2006 en el marco del Programa de Posgrado en Ciencias de la Atmósfera de la Universidad de Costa Rica. Los autores desean agradecer al Dr. Luis Farfán y al Dr. Eric Alfaro por sus valiosos comentarios. Para la realización de este trabajo se contó con el apoyo financiero por parte del proyecto Variabilidad climática, modelado y predicción de hidroclimatología regional (UCR-VI-805-98-506) y de National Oceanic and Atmospheric Administration Office for Global Programs (NOAA/OGP) y del Comité Regional de Recursos Hidráulicos $(\mathrm{CRRH})$, por medio de un proyecto regional para modelado climático. Agradecimiento especial a los proyectos ANUIES/CSUCA y Assessments of Impacts and Adaptations to Climate Change (AIACC-LA06). A las siguientes instituciones: Centro de Investigación Científica y de Educación Superior de Ensenada (CICESE), Instituto Panamericano de Geografía e Historia (IPGH), International Research Institute for Climate and Society (IRI), Centro de Ciencias de la Atmósfera (CCA) de la Universidad Nacional Autónoma 
de México (UNAM) y Universidad de los Andes, Venezuela. A Blanca Calderón por su asistencia técnica. Finalmente, los autores agradecen los comentarios y sugerencias de los revisores del trabajo.

\section{Referencias}

[1] Amador, J. A.; Alfaro, E.; Lizano, O.; Magaña, V. (2006) "Atmospheric forcing of the eastern tropical Pacific: a review", Progress in Oceanography 69(2-4): 101-142.

[2] Arakawa, A.; Schubert, W. (1974) "Interaction of a cumulus cloud ensemble with the large scale environment: Part I", J. Atmos. Sci. 31: 674-701.

[3] Castro, C. L.; Pielke Sr., R. A.; Leoncini, G. (2005) "Dynamical downscaling: an assessment of value retained and added using the Regional Atmospheric Modeling System (RAMS)", J. Geophys. Res. 110 doi:10.1029/2004JD004721.

[4] Chase, T. N.; Pielke Sr., R. A.; Castro, C. (2003) "Are present day climate simulations accurate enough for reliable regional downscaling?", Water Resources Update 124: $26-34$.

[5] Cox, R.; Bauer, B. L.; Smith, T. (1998) "A mesoscale model intercomparison", Bull. Amer. Meteor. Soc. 79: 265-283.

[6] Dickinson, R. E.; Errico, R. M.; Giorgi, F.; Bates, G. T. (1989) "A regional climate model for Western United States", Clim. Chang. 15: 383-422.

[7] Dudhia, J. (1989) "Numerical study of convection observed during the winter monsoon experiment using a mesoscale two-dimensional model", J. Atmos. Sci. 46: $3077-3107$.

[8] Dudhia, J.; Gill, D.; Guo, Y.-R.; Manning, K.; Wang, W.; Bruyere, C. (2005) "PSU/NCAR mesoscale modeling system tutorial class notes and users' guide: MM5 modeling system version 3", Mesoscale and Microscale Meteorology Division, National Center for Atmospheric Research, Boulder, Colorado.

[9] Duffy, P. B.; Govindasamy, B.; Milovich, J.; Taylor, K.; Wehner, M.; Lamont, A.; Thompson, S. (2003) "High resolution simulations of global climate. Part I: Present climate", Clim. Dyn. 21: 371-390.

[10] Gill, D. O. (1992) "A user's guide to the Penn State/NCAR mesoscale modeling system", NCAR Technical Note 381+IA.

[11] Giorgi, F. (1990) "Simulation of regional climate using a limited area model nested in general circulation model" J. Climate 3: 941-963.

[12] Giorgi, F.; Bates, G. T. (1989) "The climatological skill of a regional model over complex terrain", Mon. Wea. Rev. 117: 2325-2347. 
[13] Grell, G. A.; Kuo, Y. H.; Pasch, R. (1991) "Semi-prognostic tests of cumulus parameterization schemes in the middle latitudes", Mon. Wea. Rev. 119: 5-31.

[14] Grell, G. A.; Dudhia, J.; Stauffer, D. R. (1993) "A description of the fifth generation Penn State/NCAR mesoscale model", NCAR Technical Note 398+IA, National Center for Atmospheric Research, Boulder, Colorado.

[15] Hesselbjerg, C. J. (2000) "Use of regional climate models in regional scenario construction", in: S. Planton, C. Hanson, D. Viner D., M. Hoepffner (Eds.) ECLAT-2 Workshop, Keynote paper No. 1, Report No. 4, Climatic Research Unit, UEA, 25-27 October 2000, Toulouse, France: 26-37.

[16] Huth, R.; Metelka, L.; Kliegrová, S.; Sedlák, P.; Kyselý, J.; Mládek, R.; Halenka, T.; Kalvová, J. (2001) "Regional climate model Aladin-Climate - A tool for regionalization of climate change estimates in Central Europe: first results", in: 150 Years of Meteorological Service in Central Europe [CD-ROM], Stará Lesná, Slovakia: 9 pp.

[17] Huth, R.; Mládek, R.; Metelka, L; Sedlák, P.; Huthová, Z.; Kliegrová, S.; Kyselý, J.; Pokorná, L.; Halenka, T.; Janousek, M. (2003) "On the integrability of limitedarea numerical weather prediction model Aladin over extended time periods", Stud. Geophys. Geod. 47: 863-873.

[18] Jones, R.G.; Murphy, J. M.; Noguer, M. (1995) "Simulation of climate change over Europe using a nested regional-climate model. Part I: assessment of control climate, including sensitivity to location of lateral boundaries", Q. J. R. Meteor. Soc. 121: 1413-1449.

[19] Kain, J. S.; Fritsch, J. M. (1993) "Convective parameterization for mesoscale models: the Kain-Fritsch scheme. The representation of cumulus convection in numerical models", Meteor. Monogr. 24: 165-170.

[20] Kuo, H. L. (1974) "Further studies of the parameterizations of the influence of cumulus convection on large scale flow", J. Atmos. Sci. 31: 1232-1240.

[21] Leung, L. R.; Mearns, L. O.; Giorgi, F.; Wilby, R. L. (2003) "Regional climate research: needs and opportunities", Bull. Amer. Meteor. Soc. 84: 89-95.

[22] Pope, V. D.; Stratton, R. A. (2002) "The processes governing horizontal resolution sensitivity in a climate model", Clim. Dyn. 19: 211-236.

[23] Qian, J. H.; Seth, A.; Zebiak, S. (2003) "Reinitialized versus continuous simulations for regional climate downscaling", Mon. Wea. Rev. 131: 2857-2874.

[24] Rivera, E. R.; Amador, J. A. (2008): "Predicción estacional del clima en Centroamérica mediante la reducción de escala dinámica. Parte I: Evaluación de los modelos de circulación general CCM3.6 y ECHAM4.5", aceptado en Revista de Matemática: Teoría y Aplicaciones. 
[25] Silva, Y. (2000) "Descripción del modelo de meso-escala PSU/NCAR MM5", en: Primer Seminario de Modelaje Numérico, Oceánico, Atmosférico, Hidrológico y Biológico, 16 de agosto de 2000, Instituto Geofísico del Perú, Lima, Perú.

[26] Takle, E. S.; Gutowski Jr., W. J.; Arritt, R. W.; Pan, Z.; Anderson, C. J.; Ramos da Silva, R.; Caya, D.; Chen, S.-C.; Giorgi, F.; Christensen, J. H.; Hong, S.-Y.; Juang, H.-M.; Katzfey, J.; Lapenta, W. M.; Laprise, R.; Liston, G. E.; Lopez, P.; McGregor, J.; Pielke Sr., R. A.; Roads, J. O. (1999) "Project to intercompare regional climate simulations (PIRCS): description and initial results", J. Geophys. Res. 104: 19443-19461.

[27] Walsh, K.; McGregor, J. L. (1995) "January and July climate simulations over the Australian region using a limited-area model", J. Climate 8: 2387-2403.

[28] Wang, Y.; Leung, L. R.; McGregor, J. L.; Lee, D.-K.; Wang, W.-C.; Ding, Y.-H.; Kimura, F. (2004) "Regional climate modeling: progress, challenges and prospects", J. Meteor. Soc. Japan 82(6): 1599-1628.

[29] Wilby, R. L.; Dawson, C. W. (2001) "Using SDSM - A decision support tool for the assessment of regional climate change impacts", Environmental Modelling 8 Software 17(2): 145-157.

[30] Wilks, D. S. (1995) Statistical Methods in the Atmospheric Sciences. Academic Press, New York.

[31] Zhang, D. L.; Anthes, R. A. (1982) "A high-resolution model of the planetary boundary layer sensitivity tests and comparisons with SESAME-79 data", J. Appl. Meteor. 21: 1594-1609. 